Vol. 14, $\mathbf{N}^{\circ}$ 1-2 | 1er semestre 2020 - 2ème semestre 2020

Mutations numériques de la musique : des

contradictions à analyser

\title{
Changement technologique et sociabilité : les trois âges des communautés virtuelles africaines
}

Technological change and sociability: the three ages of African virtual communities

Cambio tecnológico y sociabilidad: las tres edades de las comunidades virtuales africanas

\section{Gado ALZOUMA}

\section{(2) OpenEdition} Journals

\section{Édition électronique}

URL : http://journals.openedition.org/ticetsociete/5222

DOI : 10.4000/ticetsociete.5222

\section{Éditeur}

Association ARTIC

Édition imprimée

Pagination : 273-302

\section{Référence électronique}

Gado ALZOUMA, « Changement technologique et sociabilité : les trois âges des communautés virtuelles africaines », tic\&société [En ligne], Vol. 14, № 1-2 I 1er semestre 2020 - 2ème semestre 2020, mis en ligne le 11 novembre 2020, consulté le 24 février 2021. URL : http://journals.openedition.org/ ticetsociete/5222 ; DOI : https://doi.org/10.4000/ticetsociete.5222 
tic\&société - 14(1-2), 2020

Changement technologique et sociabilité : les trois âges des communautés virtuelles africaines

\section{Gado ALZOUMA}

Gado Alzouma est professeur titulaire en anthropologie et sociologie. Il enseigne actuellement à l'Université américaine du Nigeria. II est l'auteur de nombreux travaux consacrés aux technologies de l'information de la communication en Afrique.

alouzma@aun.edu.ng 
Changement technologique et sociabilité : les trois âges des communautés virtuelles africaines

\title{
Changement technologique et sociabilité : les trois âges des communautés virtuelles africaines
}

Résumé : Dans cet article, nous nous proposons d'examiner les multiples facettes des liens qui se forment entre usagers du numérique en Afrique au travers de l'évolution des communautés virtuelles sur le continent au cours des 25 dernières années. Les deux principales questions ayant guidé cette recherche sont les suivantes: quels sont les différents types de communautés virtuelles auxquels les pratiques et l'appropriation du numérique ont donné lieu en Afrique? Comment sont affectées les structures générales de ces communautés, leurs configurations, lorsque le type d'objet technique qu'elles utilisent pour se fédérer change au cours du temps ? Pour répondre à ces questions, nous accorderons une attention particulière aux changements ayant affecté la technologie et aux nouvelles formes de sociabilité rendues possibles par l'usage de nouvelles applications, notamment les réseaux sociaux et les services de messagerie tels que WhatsApp, mais aussi à la naissance d'une culture numérique africaine qui se manifeste par la croissance exponentielle des applications indigènes, la création des startups africaines, ainsi que la colonisation de l'imaginaire africain par le numérique dans les domaines de l'art et des activités ludiques.

Mots-clés: Afrique, communautés virtuelles, sociabilités, réseaux sociaux, identités.

\section{Technological change and sociability: the three ages of African virtual communities}

\begin{abstract}
In this article, we examine the multiple facets of the links created over the past 25 years among digital users as they participate in African virtual communities. The two main questions that guided this research are: What different types of virtual communities have digital practices and appropriation produced in Africa? How do the general structures of these
\end{abstract}


communities and their configurations change over time with the evolution of the federating technical object? To answer these questions, we will pay particular attention to technical changes and to the new forms of sociability made possible by the use of innovative applications, particularly social networks and messaging services such as WhatsApp, as well as to the emergence of an African digital culture that shows itself in the exponential growth of indigenous applications, the creation of African startups, and the colonization of the African imaginary by digital representations in art and leisure activities.

Keywords: Africa, virtual communities, sociabilities, social networks, identities.

\title{
Cambio tecnológico y sociabilidad: las tres edades de las comunidades virtuales africanas
}

\begin{abstract}
Resumen: Este artículo examina las múltiples facetas que caracterizan a los vínculos que se están formando entre los usuarios digitales en África, mediante el estudio de la evolución de las comunidades virtuales en el continente africano en los últimos 25 años. Las dos principales preguntas que han guiado esta investigación son las siguientes: ¿Cuáles son los diferentes tipos de comunidades virtuales que se han originado en África, a través de las prácticas y de la apropiación digital? ¿Cómo se ven afectadas las estructuras generales y las configuraciones de estas comunidades, cuando el tipo de objeto técnico que utilizan para relacionarse cambia con el tiempo? Para responder a estas preguntas, prestaremos especial atención a los cambios tecnológicos y a las nuevas formas de sociabilidad que permiten el uso de nuevas aplicaciones, en particular las redes sociales y los servicios de mensajería como WhatsApp. También se analiza el nacimiento de una cultura digital africana que se caracteriza por el crecimiento exponencial de las aplicaciones indígenas, por la creación de nuevas empresas africanas, y por la colonización del imaginario africano por lo digital en los campos del arte y del entretenimiento.
\end{abstract}


Changement technologique et sociabilité : les trois âges des communautés virtuelles africaines

Palabras claves: África, comunidades virtuales, Ssociabilidad, redes sociales, identidades. 


\section{Introduction}

Alors que les recherches consacrées aux technologies de l'information et de la communication (TIC), notamment en Afrique, sont nombreuses et croissantes, elles se sont pour l'essentiel limitées à l'examen d'un certain nombre de thèmes fondamentaux tels que ceux liés au développement économique (Mansell et When, 1998 ; UNDP, 2001 ; Waverman, Meschi et Fuss, 2005), à l'accès et à la fracture numérique (Conte, 2001 ; Sagna, 2006), aux usages (Gabas, 2005 ; Chéneau-Loqay, 2010) et, pour quelques autres dont le champ d'investigation va au-delà de l'Afrique, aux inégalités sociales (DiMaggio et Hargittai, 2001; Van Dijk, 2005 : Witte et Mannon, 2010). Ce n'est pas l'importance (unanimement reconnue) de ces travaux fondateurs qui est en cause ici, mais l'orientation qu'ils ont contribué à donner aux recherches consacrées au continent dans le domaine des TIC, notamment chez les auteurs africains.

En effet, comme pour tout ce qui concerne les investigations scientifiques en Afrique, le règne sans partage du paradigme développementaliste a contribué à négliger certaines des questions relatives aux TIC qui, dans d'autres contextes sociaux, ont occupé une place importante dans la littérature afférente. C'est notamment le cas de la question des communautés virtuelles africaines à laquelle relativement peu de chercheurs (si l'on exclut Bastian, 1999 ; Damome, 2012 ; Donsimoni, 2018 ; Kiyindou et Miéré Pélage, 2012 ; Ojo, 2005 ; Traoré, 2012) se sont intéressés sur le continent. Une des premières explications à ce manque d'intérêt réside sans doute dans le fait que l'accès à Internet était relativement limité en Afrique, du moins jusqu'à l'avènement de l'Internet mobile et des réseaux sociaux. Une seconde explication peut aussi être trouvée dans l'inhibition théorique si caractéristique de la recherche africaine, notamment pour tout ce qui concerne les changements sociaux ou l'avènement de nouveaux groupes et de nouvelles formes de sociabilité (il faut songer, par exemple, à l'absence presque totale d'études sur le capitalisme africain, sur les classes sociales ou les inégalités sociales de classes en Afrique et au nombre incommensurablement plus élevé de recherches sur 
Changement technologique et sociabilité : les trois âges des communautés virtuelles africaines

" les projets de développement » ou " la corruption »). Pourtant, la question des communautés virtuelles africaines est liée à certains des aspects les plus importants de la culture matérielle africaine telle qu'elle se manifeste aujourd'hui. Dans cette perspective, il est peut-être temps que les chercheurs en sciences sociales (notamment ceux qui s'intéressent à l'étude social de la technologie) commencent à accorder une attention plus soutenue à la culture numérique africaine, c'est-à-dire à ce qu'il y a de spécifique dans les usages sociaux des technologies de l'information et de la communication en Afrique, usages qui portent sans doute la marque des cultures africaines et sont l'expression de représentations culturelles de la technique telles qu'elles se manifestent (de diverses façons) sur ce continent.

C'est pourquoi, dans cet article, nous nous proposons d'examiner les multiples facettes des liens qui se forment entre usagers du numérique en Afrique au travers de l'évolution des communautés virtuelles sur le continent au cours des vingt-cinq dernières années. Nous avons à cet effet identifié trois générations de communautés virtuelles africaines.

La première génération correspond à l'avènement des Usenet, des litservs et autres forums de discussion en ligne. Comme divers autres groupes de populations dans le monde, la diaspora africaine s'est servie de ces outils pour créer des communautés en ligne qui avaient, pour la plupart, un caractère essentiellement identitaire. Elles manifestaient, de notre point de vue, ce que Benedict Anderson (1991) a appelé le «longdistance nationalism », c'est-à-dire un ensemble d'idées et de pratiques (qui ont pour support les TIC) qui relient des individus issus d'un territoire qu'ils identifient comme un foyer ancestral et qui sont dispersés à travers le monde. Ces communautés correspondaient non seulement aux premières formes d'appropriation du Net par les Africains, mais encore à une " tribalisation ", une " ethnicisation », voire une " racialisation » du Net qui s'est manifestée par la création de sites ethniques et de sites panafricains.

La seconde génération correspond à l'abandon progressif des sites de discussion en ligne et à la migration des communautés virtuelles africaines sur les réseaux sociaux et les services de messagerie en ligne qui ont pour support le téléphone mobile. Ce mouvement correspond à l'éclosion de groupes plus divers, moins englobants et qui se déplacent des perspectives 
nationales ou panafricaines (sans les abandonner) vers des perspectives plus communautaires et la création de groupes sur les réseaux sociaux à une échelle plus réduite, tels que les groupes de professionnels travaillant dans le même domaine ou la même compagnie, les groupes d'étudiants des mêmes universités, les groupes de ressortissants de la même région, voire du même village sur WhatsApp, ou des groupes familiaux, des groupes d'amis, etc. En même temps, l'appartenance des individus à ces groupes devient plurielle dans la mesure où le même individu peut être membre de multiples réseaux sociaux et de multiples groupes sur WhatsApp. Les perspectives identitaires, qu'elles soient ethniques, tribales ou panafricaines, n'ont pas disparu pour autant et se manifestent dans la " communautarisation » du web sur lequel de nombreux groupes ont créé des pages sur Facebook et se servent du téléphone portable et des services de messagerie comme WhatsApp pour des objectifs de mobilisation religieuse à l'échelle nationale ou continentale ou des objectifs de mobilisation et de coordination politiques qui permettent aux communautés diasporiques de prendre part aux débats nationaux et d'altérer, voire de fortement influencer la direction des affaires politiques nationales, le discours politique et les prises de décision majeures dans leurs pays respectifs. Un exemple récent est la déclaration d'indépendance de la partie occidentale du Cameroun, connue sous le nom d'Ambazonia et dont l'existence était toute virtuelle avant de se transférer sur le terrain du combat politique.

La troisième génération voit l'avènement d'une croissance très rapide de création d'applications et de contenus proprement africains à la différence du premier âge du web, durant lequel non seulement le langage d'Internet était étranger (européen) mais les contenus mis en ligne avaient pour origine des sites qui n'étaient pas africains. Toutefois, la spécificité de cette troisième génération de communautés virtuelles ne tient pas qu'à la création de contenus proprement africains. Elle tient surtout au fait qu'en dehors de l'utilisation massive de Facebook, de Twitter, d'Instagram ou de WhatsApp, de très nombreux réseaux sociaux sont en train d'être créés localement par les Africains euxmêmes et que ceux-ci rencontrent, sur le continent, autant ou encore plus de succès que Facebook, par exemple. Ainsi, Mxit en Afrique du Sud compte des dizaines de millions de membres, et l'adhésion massive à d'autres réseaux sociaux tels qu'Eskimi ou Yookos au Nigeria, ou encore Esoko au Ghana, par exemple, 
Changement technologique et sociabilité : les trois âges des communautés virtuelles africaines

et à de nombreux autres ailleurs en Afrique est un phénomène nouveau qui ne se réduit pas à la « domestication " exotique de ces objets techniques ou à des «modes d'appropriation innovants " (autre nom donné à l'exotisme) tels qu'une partie de la littérature concevait l'appropriation du téléphone portable il y a encore quelques années. Si on ajoute à cela l'explosion des startups africaines, on est obligé de conclure qu'il s'agit d'un phénomène nouveau qui, peut-être, fera passer les Africains de récepteurs et concepteurs passifs de TIC à celui de producteurs d'objets et de sens.

Toutefois, il ne faut pas voir entre ces trois générations de communautés virtuelles africaines une ligne de démarcation nette ou croire que l'existence de l'une est exclusive de l'autre, ou même supposer que la vieille génération doit d'abord disparaître avant que naisse la nouvelle. Ces trois générations coexistent, se chevauchent ou s'enchevêtrent bien souvent dans certains de leurs segments bien qu'on observe que l'importance des plus vieilles tend à décroître et que les plus nouvelles tendent à avoir beaucoup plus de succès au cours du temps et a à se substituer progressivement aux anciennes.

\section{Méthodologie}

Sur le plan méthodologique, il faut d'abord souligner que notre objectif était d'identifier les changements majeurs (qu'on peut qualifier de "tournants») ayant marqué l'évolution de communautés virtuelles africaines. Pour ce faire, nous avons d'abord eu recours à une recherche documentaire portant sur les études qui ont été consacrées à ces communautés virtuelles puis à une recherche et à une identification des sites web les plus représentatifs à cet effet. Pour juger du degré de " représentativité " des sites web en question, nous nous en sommes tenu à l'importance que leur ont accordée les chercheurs qui les ont mentionnées dans leurs études. II ne s'agit donc pas d'un recensement quantitatif ou exhaustif qui a pour but de dégager un échantillon représentatif, mais bien plutôt d'une appréhension qualitative de productions symboliques virtuelles au regard de leur importance "historique " ou "généalogique " attestée par le fait qu'elles ont retenu l'attention de chercheurs et sont emblématiques des communautés créées en ligne par les Africains. Cette démarche 
a l'avantage de circonscrire le champ de la recherche à un nombre relativement restreint de sites web et de rendre en conséquence possible une analyse à l'échelle de l' « Afrique » qui autrement se serait révélée fastidieuse.

C'est dans ce même ordre d'idées que nous nous sommes limité à présenter quelques exemples de pays africains suffisamment représentatifs des processus en cours, entre autres le Nigeria, le Kenya et, pour ce qui est des pays francophones, les communautés virtuelles de la diaspora africaine établie en France. Nous sommes conscient des limites de cette recherche, notamment dans son ambition " englobante », mais cette démarche est moins dictée par un choix délibéré de l'auteur que par la façon dont ces communautés se sont historiquement formées; elles sont d'abord nées dans la diaspora avant de naître dans des pays africains spécifiques. Elles ont aussi souvent revêtu un caractère " panafricain » avant de devenir des sites "nationaux ", ceci étant le résultat du développement et de l'appropriation des technologies de l'information et de la communication dans les pays occidentaux qui se sont plus tard transposés en Afrique. Ces communautés virtuelles étaient donc "africaines » ou "diasporiques" avant de devenir "sénégalaises", " nigériennes », " camerounaises », etc. En conséquence, leur recension généalogique a dû épouser cet ordre d'apparition historique.

\section{Qu'est-ce qu'une communauté en ligne?}

Bien que son emploi soit quelque peu "lourd » en français, certains auteurs (Boutet, 2008; Gensollen, 2006 ; MercantiGuérin, 2010) préfèrent utiliser la notion de communauté en ligne plutôt que celle de communauté virtuelle ou de cybercommunauté, suivant en cela la tradition anglo-saxonne, où la notion de online communities est plus utilisée aujourd'hui que celle, plus vielle, de virtual communitities ou de cybercommunities. Comme souligné par Serge Proulx (2006), cette préférence a sans doute quelque chose à voir avec les connotations attachées à la notion de virtualité, qui soulignent l'absence d'une chose alors que ce à quoi nous avons à faire ici (les TIC) se manifeste par des usages, des pratiques, des réseaux, ainsi que par des objets et leur manipulation, qui sont 
Changement technologique et sociabilité : les trois âges des communautés virtuelles africaines

tout ce qu'il y a de réel. Toutefois, si le caractère virtuel des espaces et des communautés dont il est question peut sembler " déplacé », les groupes auxquels cet adjectif est accolé ont aussi fait l'objet de nombreux débats consistant à savoir si les noms par lesquels nous les désignons et qui sont ceux tirés de l'expérience de la vie quotidienne, du monde social " réel », sont appropriés. Est-il juste de dire qu'il s'agit de "communautés »? En quoi ont-ils les caractères des communautés telles que nous les définissons? II serait fastidieux de revenir sur tous ces débats menés depuis le début des années 1990 jusqu'au milieu des années 2000 sur ces sujets, mais nous soulignerons essentiellement les raisons pour lesquelles la notion même de communauté virtuelle (ou de communauté en ligne) a fini par s'imposer comme un concept opérationnel dans le domaine des théories de la communication.

La première raison est l'existence de "liens » (Proulx, 2006) entre ceux qui forment ces groupes, c'est-à-dire l'existence d'interactions, à la façon dont les sociologues différencient le groupe social de l'agrégat, par l'existence d'échanges entre ses membres. II existe en effet, entre les membres d'une communauté virtuelle, des échanges au moyen de systèmes de symboles qui peuvent revêtir divers caractères (écrit, audio, visuel, verbal). Ces liens qui se créent au travers des échanges peuvent être durables et susciter de l'affection ou l'idée d'intérêts partagés en commun et de pratiques communes (auquel cas on parle de "communautés d'intérêt » ou de "communautés de pratique »), que ceux-ci aient préalablement existé dans le monde réel pour ensuite se traduire par la création de groupes en ligne, ou que le groupe en ligne soit le premier et le seul lien existant entre les membres qui le composent, ou, enfin, que le groupe en ligne se manifeste dans le monde réel par un groupe réellement existant, un groupe dont il a contribué à rendre l'existence possible. II faut songer, dans ce dernier exemple, aux printemps arabes et aux mobilisations politiques rendues possibles par l'usage du téléphone portable, de Twitter, de Facebook et de nombreux autres réseaux sociaux.

La deuxième raison a trait à l'existence d'objets et d'objectifs qui sont aussi ceux qui motivent la création de communautés dans le monde réel, par exemple les communautés ludiques, sportives, politiques ou les groupes de parenté, de voisinage, de résidence, de genre, d'âge, ou même les nations, les 
communautés religieuses, les communautés morales transnationales qui luttent pour promouvoir des valeurs universelles et dont les membres se recrutent sur tous les continents, dans tous les pays, dans toutes les "races ", etc. Toutes ces communautés du monde réel ont leurs pendants virtuels. Cependant, contrairement à l'idée répandue au début des années 2000, empressons-nous de dire que ces communautés n'ont pas disparu pour céder la place à leurs " avatars" sur Internet, comme le suggérait par exemple Castells (2000), qui écrivait: "Une fois introduites avec pour support les technologies de l'information, les réseaux d'information, par la concurrence, éliminent progressivement d'autres formes organisationnelles enracinées dans une logique sociale différente. En ce sens, ils tendent à affirmer la prédominance de la morphologie sociale sur l'action sociale ${ }^{1}$ " (p. 16). Rien de tel ne semble s'être concrétisé à ce jour. En revanche, il est indéniable que les groupes existants ont migré sur Internet ou s'y sont simplement transposés. On note aussi le fait que des groupes qui n'existaient pas auparavant ont été rendus possibles par l'avènement d'Internet. Nous ne devons donc pas oublier les limites du déterminisme technologique.

Enfin, la troisième raison est l'existence d'un espace de sociabilité. La proximité physique de ses membres était l'un des aspects essentiels (pas exclusifs) par lesquels on définissait autrefois la communauté. II faut, par exemple, songer aux notions de terroirs, voire de territoires, d'espace de vie, d'espace « local », d'espace commun, d'espace public, d'espace villageois, de lieux de sociabilité, d'espace partagé, etc., bref toutes ces notions liées à l'espace et qui évoquent invariablement l'ancrage d'une communauté dans un emplacement partagé ou des sites d'échanges communautaires auxquels s'identifient des individus au travers d'attaches multiples. Dans le même ordre d'idées, on peut estimer qu'une communauté virtuelle a une " niche » qui est le réceptacle et le lieu de production et de reproduction d'un ensemble continu de liens sociaux et symboliques qui tiennent les membres ensemble et les distinguent des autres.

1 Traduction libre de: « Once introduced, and powered by information technology, information networks, through competition, gradually eliminate other organizational forms, rooted in a different social logic. In this sense, they tend to assert the predominance of social morphology over social action ». 
Changement technologique et sociabilité : les trois âges des communautés virtuelles africaines

C'est tout cela qui fait des communautés virtuelles des groupes qui partagent toutes les caractéristiques par lesquelles nous définissons ordinairement les communautés dont nous sommes membres. Ces communautés, comme tous les groupes, sont affectées par les changements qui traversent les sociétés, notamment dans leur dimension matérielle et technologique. Leur existence manifeste la façon dont les usagers s'organisent pour faire face à l'évolution rapide des technologies (dans leurs fonctionnalités, leurs interfaces, leurs plateformes, leurs applications et leurs accès) et pour préserver, renforcer ou maintenir les liens qui les unissent dans des configurations adaptées à la situation nouvelle. C'est ainsi qu'il est possible d'identifier trois types de communautés virtuelles africaines qui correspondent aussi à trois âges du numérique en Afrique.

\section{Les communautés virtuelles africaines de première génération}

Les premières communautés virtuelles africaines sont apparues sur des forums de discussion en ligne (notamment Usenet, précurseur des "médias sociaux " en ligne, établi depuis 1980) ou des litservs (listes de diffusion électroniques basées sur l'échange de courriels et permettant d'envoyer des messages à plusieurs abonnés en même temps). La sociabilité étant un besoin humain fondamental, on pouvait s'attendre à ce que les Africains saisissent l'opportunité de l'existence de ces technologies pour créer des communautés virtuelles.

À cet égard, il est difficile de préciser à quel moment sont nées les premières communautés virtuelles africaines, mais il ne fait pas de doute que, dès la fin des années 1990 et au tout début des années 2000 , une littérature croissante a commencé à y faire référence. Nous pensons notamment aux travaux de Hall (1998), de Bastian (1999), de Moran (2000), de Darkwa et Mazibuko (2000) et, de façon encore plus explicite et exclusive, à un article d'Ojo daté de 2005. Ces travaux permettent de montrer que l'Afrique du Sud et la diaspora africaine peuvent être considérées comme ayant joué le rôle de pionniers dans la création des cybercommunautés africaines. En effet, dès 1999, Bastian faisait remarquer que 


\section{Gado ALZOUMA}

les immigrés nigérians du monde entier qui ont accès à des ordinateurs personnels avec des modems ou des postes de travail connectés aux réseaux locaux entrent de plus en plus dans le cyberespace et construisent ce que j'appelle un " Nigeria virtuel » grâce au email, une efflorescence de sites web avec un contenu nigérian et, en particulier, une liste de diffusion (qui transcende les frontières, comme je le mentionnerai plus loin) appelée « Naijanet $»^{2}$. (p. 15.)

Selon des informations recueillies par Bastian auprès du premier administrateur du réseau, la liste de diffusion Naijanet date de 1991, lorsque des membres de la diaspora nigériane ont commencé à échanger des courriels relatifs à des questions nigérianes. Ce système d'échanges informels a ensuite été transformé en un véritable réseau social électronique dédié à des discussions sur les problèmes sociaux et politiques relatifs au Nigeria. Son succès a été tel qu'entre 1996 et 1997, Naijanet comptait déjà 630 membres (Bastian, 1999). Dans le sillage de Naijanet, de nombreux autres réseaux sociaux vont être créés. On peut noter, parmi ceux-ci, soc. culture. nigeria créé sur Usenet (le système de discussion électronique à caractère global qui existe encore aujourd'hui, mais qui a été particulièrement actif entre 1980 et 2011). Moran qui, en 2000, a conduit une étude sur soc. culture. nigeria écrit notamment à ce sujet : "Les Nigérians de la diaspora qui écrivent sur soc. culture. nigeria décrivent des visites régulières à la maison, postulent pour des emplois au Nigeria, téléphonent régulièrement et cherchent à s'y marier $^{3}$. » (p. 15.)

D'autres initiatives dans d'autres pays africains ont immédiatement suivi celles du Nigeria, notamment KenyaNet (Kenya, ancêtre d'AfricaOnline, créé en 1994), TanzazaNet (Tanzanie), BurundiNet (Burundi), etc. On peut considérer ces listes de diffusion comme les premières startups indigènes en Afrique, car elles ont été créées en Afrique par des Africains. Si

\footnotetext{
2 Traduction libre de : «Nigerian immigrants throughout the world who have access to personal computers with modems or office workstations connected to local area networks are entering into cyberspace in increasing numbers and are constructing what I call a "virtual Nigeria" through the use of email, an efflorescence of websites with Nigerian content and particularly a listserv (that transcends the boundaries, as I will discuss below, of a single list) called Naijanet. "

3 Traduction libre de: "Nigerians in diaspora writing on soc.culture.nigeria describe regular visits home, apply for jobs in Nigeria, telephone regularly and seek to marry there ".
} 
l'on prend l'exemple de Tanzanet (Tanzanian Electronic Networking Community), elle se décrit comme «la première organisation virtuelle en Tanzanie » et se donne pour objectif de «fournir un forum pour l'échange d'informations entre les ressortissants tanzaniens et d'autres personnes intéressées ${ }^{4}$ ॥ à travers le monde. Ses origines remontent à 1992, année à partir de laquelle elle a grandi pour atteindre jusqu'à 400 membres en 1995. Comme toutes les listes de diffusion de première génération, elle existe encore aujourd'hui, mais n'est plus aussi active qu'elle l'était jusqu'au milieu des années 2000.

Pour ce qui est de l'Afrique francophone, le premier groupe de discussion qui avait un caractère panafricain est probablement le forum de discussion francophone africain créé sur AfricaOnline. AfricaOnline est un fournisseur de services Internet africain créé en 1994 par des Kenyans et qui a permis l'éclosion de nombreux forums où les nationaux de divers pays africains pouvaient créer des groupes relatifs à leur pays ou discuter de sujets à caractère continental. Par la suite, au début des années 2000 , trois sites principaux vont dominer la scène des communautés virtuelles francophones : Afrik.com, qui a existé à partir de 2000 et qui avait un forum de discussion qui a fonctionné au moins jusqu'en 2003; puis Afrikara.com et Grioo.com, créés en 2004 ; et Africamaat.com. Bien entendu, cette liste ne prétend pas à l'exhaustivité et de nombreux autres forums dont nous n'avons pas connaissance ont existé ou nous ne les mentionnons pas ici. Par ailleurs, on ne saurait réduire ces communautés virtuelles aux seuls forums de discussion. Des communautés virtuelles africaines d'autres natures existaient déjà ${ }^{5}$. Toutefois, il nous semble que les forums de discussion

\footnotetext{
${ }^{4}$ Traduction libre de : «TANZANET - the Tanzanian Electronic Networking Community is the first and almost certainly the most successful 'virtual organization' in Tanzania...TANZANET is a world-wide discussion non-profit Organization whose primary objective is to provide a forum for information exchange among Tanzanian nationals and other interested persons through the world-wide Internet. " (http://www.tanzanet.org/)

${ }^{5}$ On peut noter, par exemple, le site d'Africsearch, un cabinet de recrutement et de conseils en ressources humaines daté de 1996 et qui a maintenant acquis une grande reconnaissance internationale. On peut également noter les très nombreux sites de recherche d'emplois ou des sites comme ExcelAfrica, qui se décrit comme le portail de l'éducation internationale africaine. On peut enfin citer les innombrables sites web communautaires tels que les sites de rencontres, les sites dédiés à l'actualité de l'Afrique et de la diaspora noire, les sites web des multiples associations, les sites de divertissement et d'art, les sites dédiés à certaines catégories sociales telles que les femmes noires, les blogues et les pages personnelles d'activistes influents, de politiciens, d'écrivains et de leaders religieux, etc. Nous avons également l'existence
} 


\section{Gado ALZOUMA}

étaient les plus significatifs.

De ces premières observations, on peut tirer les conclusions suivantes. Tout d'abord, les premières communautés virtuelles africaines sont nées dans la diaspora. Ceci s'explique par le fait que, dans la plupart des cas, les communautés diasporiques avaient un accès plus élevé aux TIC que les gens restés sur le continent. La seconde raison est qu'Internet s'est présenté comme une occasion, pour toutes «les communautés dispersées à travers le monde, de retrouver et partager une appartenance identitaire qu'elles croyaient parfois perdue ${ }^{6}$ » (Alzouma, 2008, p. 201). Elle a également «favorisé des reconstructions identitaires par le biais de représentations nouvelles, constamment formulées, nourries et entretenues comme un patrimoine immatériel ${ }^{7}$ "(Alzouma, 2008, p. 201). Cela transparaît dans le fait que l'on constate une prédominance des questions identitaires et politiques dans les forums de discussion africains de première génération. Ces premières communautés virtuelles africaines sont le plus souvent des " nations numériques » qu'on peut appréhender et analyser comme les "imagined communities" de Benedict Anderson (1991), et encore plus précisément, tel que nous l'avons souligné plus haut, comme manifestant ce que cet auteur a appelé « longdistance nationalism » (Anderson, 1991).

D'après Anderson (1991), "les deux axes cruciaux du capitalisme [...] semblent aussi être dans les transports et dans les communications ${ }^{8}$ » (p. 7), et il cite « la propagation rapide des

de nombreuses associations et organisations noires qui sont centrées sur les noirs et les intérêts des noirs. Tel est le cas, entre autres, de l'UCN7, du CRAN, du CapD 'iv8, de l'Africagora9, etc. Comme nous l'avons démontré ailleurs, l'existence de l'Internet a rendu possible la création de ces groupes virtuels qui ont en commun le fait d'être " noirs " ou " africains » et qui développent, à travers le Net, des relations et des réseaux qui sont basés sur cette réalité. Ces groupes sont des communautés parce que nous y trouvons l'expression d'intérêts communs, des interactions durables entre leurs membres et une identification commune. Enfin, ces communautés ont des " frontières » reconnaissables en raison du caractère limité des chevauchements et des "mélanges " avec d'autres groupes. Les membres de ces communautés sont également unis par la façon dont ils sont reconnus et caractérisés par d'autres groupes de la société en général comme appartenant à un ensemble commun.

6 Traduction libre de : " has allowed many scattered communities across the world to rediscover and share a common identity that was believed lost ".

7 Traduction libre de: «It has also led to the reconstruction of identities by way of new representations, which are constantly being formulated, nourished, and maintained. ॥ 8 Traduction libre de : "The two crucial axes of capitalism's thrusts seem to me to be in transportation and in communications $»$. 
Changement technologique et sociabilité : les trois âges des communautés virtuelles africaines

réseaux informatiques intercontinentaux ${ }^{9} »(\mathrm{p} .7)$ parmi ces changements majeurs. Toutefois, l'un des effets les plus importants de ces changements a porté sur les conceptions de l'identité, notamment par le biais de l'ethnicisation de la politique. Ici, il faut comprendre " ethnicisation » dans le sens le plus large possible, car non seulement, pour les forums de discussion qui nous occupent, certaines des communautés nationales créées sur des bases nationales ont très vite éclaté en de multiples sites ethniques, voire tribaux, mais des communautés diasporiques continentales se sont retrouvées sur la toile et ont fondé des groupes électroniques identitaires sur des bases parfois " raciales » (par exemple, les « Noirs de France ») ou sur la base d'une ascendance commune («les personnes d'origine africaine ») ou d'idéaux à soubassements identitaires (" ceux qui partagent les valeurs du panafricanisme »).

D'après Kiyindou (2012), en France, " parmi les sites se déclarant panafricanistes se trouvent: congopages.com, afrikadiaspora.com, afrocom.org, grioo.com, mwinda.org, afriblog.com, afriquetandem.com, mukanda.org, pratic (joretic.com), afrik.com. Ils se distinguent par leurs contenus, leurs noms, le choix des couleurs et des visuels » (p. 2). Une analyse de ces sites permet de montrer que certaines d'entre elles ont éclaté en une multitude de sites " tribaux ». C'est ainsi que la communauté nigériane qui s'était formée sur Naijanet à partir de 1991 va se scinder, dès 1992, en de multiples sousgroupes à caractère ethnique, tels qu'Oduduwa, le groupe Yoruba fondé sur le nom de l'ancêtre apical mi-dieu mi-homme connu sous le même nom. De même, un groupe Igbo, connu sous le nom d'lgbo-Net, et un autre groupe rassemblant les communautés issues de la région de Rivers, RiveNet, vont se former (voir Bastian, 1999). Le même phénomène peut être observé à propos de multiples autres pays africains où les tribus numériques ont proliféré, particulièrement en Afrique anglophone. En revanche, les sites Grioo.com, Afrikara.com et Africamaat.com, qui se sont créés en France dans la première décade des années 2000 et qui avaient tous un caractère continental, ont connu, comme nous le montrerons plus bas, une certaine désaffection avec l'avènement des réseaux sociaux. Par exemple, Grioo.com se présente comme «le site de la communauté noire de France », tandis que l'objectif avoué du

9 Traduction libre de: « The rapid spread of intercontinental computer networks ». 
site Africamaat.com et de l'institut du même nom est de valoriser « la contribution des Noirs a l'évolution scientifique ».

\section{Les communautés virtuelles de seconde génération : réseaux sociaux et services de messagerie en ligne}

La seconde décade du $21^{\text {ème }}$ siècle marque assurément un tournant pour les communautés virtuelles africaines. Si l'on en juge par le cas francophone, on peut dire que c'est à partir de ce moment-là que les forums de discussion ont commencé à enregistrer de moins en moins de membres pour devenir, pour beaucoup d'entre eux (Grioo.com, Afrikara.com, Africamaat.com, etc.), inactifs, voire totalement absents de la toile. C'est ainsi que, dans une publication intitulée « hibernation du forum », un des " grioonautes » les plus actifs du nom de Dilo faisait remarquer :

Il est dommage de constater de facto que depuis la fin de la tragédie regrettable en Côte d'Ivoire, ce forum-ci semble perdre toute ardeur. Pourtant, les opinions et apports de valeureux intervenants comme Ogo, Gnata, Samuel, et bien d'autres et leur « contrepoids » Marlvel-02, étaient non négligeables. [Maintenant ils] ont presque délaissé toute contribution, même hors du sujet sur la $\mathrm{Cl}$. Dommage! Espérons qu'ils voudront sortir ce forum panafricain du coma. (Dilo, Grioo.com, Forums, Sujet « Hibernation du forum », mercredi 30 novembre 2011, message 322)

Ce à quoi un autre "grioonaute » du nom de Merikama répondait :

Je ne crois pas du tout à une " hibernation », mais plutôt à une lente agonie vers une mort certaine. Ce phénomène n'est pas spécifique à Grioo, mais à l'ensemble des forums en général. Avec le boom des réseaux sociaux tels que Facebook et Twitter, les anciennes plateformes d'échanges d'idées que sont les forums sont devenues désuètes, du fait de leur trop grande rigidité. L'effervescence des échanges dans la communauté africaine de par le monde sur Facebook par exemple, contraste grandement avec "l'hibernation " des forums africains. Je dirais même que c'est sur Facebook qu'a lieu le plus d'échanges entre 
Changement technologique et sociabilité : les trois âges des communautés virtuelles africaines

Africains sur la toile. En ce qui me concerne, étant tellement occupé à échanger sur Facebook, c'est très rarement que je mets mon nez dans le forum de Grioo ou tout autre forum africain. (Merikama, Grioo.com, Forums, Sujet « hibernation du forum », dimanche $1^{\mathrm{er}}$ avril 2012, message 254)

L'avènement de Facebook a donc contribué à une migration des internautes africains vers les réseaux sociaux et à la disparition progressive des communautés virtuelles de première génération, ce qui donne une idée de la façon dont les groupes virtuels, à la différence des groupes du monde réel, naissent, vivent et meurent. On peut dire, en effet, que la vie des communautés virtuelles est étroitement associée à la viabilité technologique des modes d'échanges utilisés et à la pertinence de l'utilisation de l'objet technique qui devient obsolète et cesse d'être le support de l'existence d'une communauté dès lors qu'une nouvelle invention vient à le surpasser ou que des raisons d'ordre économique (par exemple, une baisse des prix ou un coût moins élevé pour une application ou un objet technique quelconque) ou social (par exemple, l'illettrisme de certains segments d'utilisateurs) justifient son abandon.

En Afrique, c'est surtout à partir de 2009 que l'utilisation de Facebook s'est répandue dans la plupart des pays africains. D'après Internet World Stats (2020), le taux de pénétration d'Internet en Afrique est désormais de 39,2\% alors que la moyenne mondiale est de $58,8 \%$ et de $62,9 \%$ pour le reste du monde, tandis que plus de 177 millions d'Africains ont souscrit à Facebook. Pour l'ensemble des réseaux sociaux, en 2017, We Are Social indiquait que le nombre d'utilisateurs s'élevait à 170 millions avec un taux de pénétration de $14 \%$. II faut aussi noter que la plupart des utilisateurs de ces réseaux sociaux étaient (et sont toujours) surtout sur Facebook, qui comptait, en 2017, 168 millions d'utilisateurs inscrits, tandis qu'Instagram, entre autres, n'en comptait que 31 millions seulement et Linkedln, 24 millions (We Are Social, 2018). Ce succès de Facebook peut surtout être expliqué par les fonctionnalités liées à la sociabilité et à une plus grande flexibilité offerte aux utilisateurs, qui peuvent non seulement créer des pages individuelles mais aussi des groupes en ligne pour des activités qui peuvent être aussi bien professionnelles que proprement sociales. Mieux encore, c'est pour ces mêmes raisons que 
WhatsApp, service de messagerie en ligne, tend de plus en plus à s'imposer dans les échanges entre Africains au détriment des messageries traditionnelles liées au téléphone portable, telles que les SMS. L'adhésion à WhatsApp croît même plus rapidement que celle à Facebook du fait que l'application semble avoir des fonctionnalités plus adaptées au contexte économique et social de l'Afrique et aux caractéristiques de la très grande majorité des usagers africains, qu'ils soient de la diaspora ou qu'ils soient restés au pays, qu'ils soient citadins ou ruraux, pauvres ou non, scolarisés ou analphabètes. Mieux encore, bien que WhatsApp soit d'abord un service de messagerie, il est semblable, par bien des aspects, à un service de réseautage social.

En effet, WhatsApp est d'abord une technologie qui « utilise la connexion internet de votre téléphone pour envoyer des message ${ }^{10} "$. Son grand intérêt, de ce point de vue, réside dans le fait qu'il permet aux utilisateurs d'échanger des messages sans avoir à payer les frais afférents aux SMS. Du point de vue du contexte africain caractérisé par la pauvreté, à cet avantage de la gratuité du coût s'en ajoutent de nombreux autres : par exemple, la possibilité de faire des appels vocaux ou des appels vidéo "face à face " (qui excluent la nécessité de savoir lire et écrire), la possibilité d'envoyer des photos et des vidéos « même avec une connexion lente ${ }^{11}$ " dans des pays où jusqu'à présent la faiblesse de la bande passante rendait aléatoire, coûteuse et extrêmement difficile l'utilisation de fonctionnalités "gourmandes» en unités d'information, telles que les téléchargements de photos et de vidéos. II faut aussi noter que WhatsApp nous donne la possibilité d'envoyer des documents, des tableaux, etc., " sans avoir à passer par les courriels ou les applications de partage de documents ${ }^{12}$ ». Enfin, les fonctionnalités de WhatsApp incluent également les chats (conversations) entre deux individus et les chats de groupes. Comme le site de ce service de messagerie le précise, n'importe qui peut créer un nombre illimité de groupes de conversation et la création d'un groupe de conversation, telle qu'elle est décrite sur le site de WhatsApp, est d'une facilité déconcertante. C'est ainsi qu'en Afrique, des groupes de toute nature sont en train

10 Présentation des fonctionnalités de WhatsApp sur https://www.whatsapp.com/fea $\frac{\text { tures/. }}{11 \mathrm{lbid} \text {. }}$

11 Ibid.
12 Ibid. 
Changement technologique et sociabilité : les trois âges des communautés virtuelles africaines

d'être créés grâce à cette technologie, que ce soit des groupes professionnels, des groupes d'amis ou des groupes fondés sur l'appartenance à la même communauté (famille, village, région, diaspora). Au Niger, par exemple, la famille élargie est l'un des groupes d'appartenance dont la création a rencontré le plus de succès sur WhatsApp (Alzouma, 2018). Les gens d'ascendance commune, qui ont perdu la mémoire de leurs relations de parenté au fil des générations par suite des dispersions ou des migrations à l'échelle nationale ou internationale, sont en train de se reconnecter et de raviver le lignage en réactivant les liens d'entraide et de solidarité intrafamiliale qui permettent de maintenir la vie du groupe. Le succès de WhatsApp est donc dû en partie au fait qu'il permet et facilite la réactivation d'espaces de sociabilité qui étaient en voie de disparition, mais dont le besoin est encore ressenti par les populations confrontées à l'anomie du monde moderne. On peut en conclure que ce média recrée des liens sociaux là où ils tendaient à être brisés, et ceci est d'une grande importance dans un continent où les forces centrifuges (terrorisme violent, notamment au Sahel, absence d'emplois pour les jeunes et migrations à grande échelle, etc.) tendent aujourd'hui à se multiplier.

La différence majeure qui existe entre ces communautés fondées sur WhatsApp et sur les réseaux sociaux et les communautés de la première génération (qui étaient essentiellement, mais non exclusivement, fondées sur les forums de discussion en ligne) réside dans l'éclatement et la diversification des buts qui, à l'origine, étaient essentiellement identitaires, politiques ou sociaux et sont devenus plus tard économiques, professionnels, ludiques et souvent, aussi, sociaux et identitaires, mais à une échelle plus réduite (famille, villages, amis, etc.). Par ailleurs la prolifération des smartphones a permis à ces communautés, qui étaient essentiellement localisées dans les diasporas en Europe et ailleurs, de migrer en Afrique, et c'est surtout dans la troisième génération, comme nous le verrons, que cette dernière tendance est en train de s'affirmer.

Enfin, les utilisateurs des réseaux sociaux en Afrique sont aujourd'hui des centaines de millions, tandis que ceux des listes de diffusion et des forums de discussion en ligne n'étaient, au début, que quelques centaines ou quelques milliers d'individus tout au plus, comme il est possible de s'en rendre compte en 
consultant le nombre d'utilisateurs qui y accédaient pour lire des postes, par exemple sur Grioo.com. Les membres de ces premières communautés virtuelles étaient aussi pour la plupart des membres de la diaspora. Ce n'est plus le cas aujourd'hui et on peut dire que la population composant les communautés virtuelles a crû par centaines de millions en moins d'une décennie, qu'elle s'est africanisée en migrant sur le continent et qu'elle se distingue de la première génération en étant surtout une communauté virtuelle adossée à l'Internet mobile, les utilisateurs étant essentiellement équipés de smartphones. Pour ces communautés, le téléphone mobile a supplanté tout autre objet technique utilisé pour communiquer. Mieux encore, la prolifération du téléphone mobile, dont le coût n'a cessé de baisser au cours des années, a rendu obsolète l'utilisation des cybercafés, qui étaient l'unique voie d'accès à Internet pour les populations pauvres d'Afrique et qui sont aujourd'hui de plus en plus désertés (Sangaré, 2017).

II semble donc qu'un " seuil critique » a été atteint aujourd'hui dans l'accès, l'appropriation et l' « indigénisation » des TIC en Afrique et que ce changement est en train de donner naissance à une troisième génération de communautés virtuelles en Afrique.

\section{Les communautés virtuelles de troisième génération}

La caractéristique majeure des communautés virtuelles de troisième génération est l'existence de plateformes numériques créées par les Africains pour les Africains. Si Facebook et WhatsApp sont des technologies globales dont les usages peuvent cependant être locaux et, pour cela, "domestiqués », les plateformes dont nous parlons ici sont des créations indigènes séparées et distinctes de celles qui sont logées sur les réseaux sociaux traditionnels. Mieux encore, certaines de ces plateformes existent depuis la première décade des années 2000 et certaines autres, telles que KenyaNet et AfricaOnline, remontent même au début des années 1990 et ont évolué en parallèle avec les sites de réseautage social les plus connus. Toutefois, la distinction principale des communautés

virtuelles de troisième génération est qu'elles ont essentiellement pour support, auprès de leur public, les 
Changement technologique et sociabilité : les trois âges des communautés virtuelles africaines

smartphones. C'est par exemple le cas de MXIT (jusqu'à sa disparition en 2015), un service de messagerie instantanée gratuit lancé en 2006 en Afrique du Sud « utilisé par plus de 100 millions de personnes dans les marchés émergents à travers le monde ${ }^{13} »$. D'après Durando et al. (2007), l'usage de MXIT était très répandu parmi les jeunes, surtout en Afrique du Sud, où cette application de messagerie instantanée mobile était la plus populaire de toutes (Chigona et Chigona, 2008). Tout comme sur WhatsApp, les utilisateurs de MXIT pouvaient créer des groupes de discussion ou avoir des échanges d'individu à individu. Les groupes de discussion peuvent être créés sur toutes sortes de bases, notamment l'amitié, le lieu de résidence, l'âge, le genre, etc. La plupart des utilisateurs utilisent des pseudonymes comme autrefois sur les forums de discussion en ligne et les numéros de téléphone qui leur servent de support n'apparaissent pas sur l'écran.

Le succès phénoménal rencontré par MXIT n'est toutefois pas isolé et on peut en citer de nombreux autres, dont les plus marquants sont par exemple Yookos, un site de réseautage social fondé en 2011, initialement à des fins de mobilisation religieuse par l'Église nigériane connue sous le nom de Christ Embassy. Yookos compte aujourd'hui plusieurs millions d'usagers à travers le monde dont les motivations et les activités ne sont plus seulement religieuses. On peut également citer le cas d'Esoko, très populaire au Ghana, surtout auprès des couches rurales. Esoko est décrit comme

un outil de communication simple qui fonctionne avec tous les modes mixtes d'applications web et mobiles, mais principalement par SMS et tout récemment sur les systèmes d'exploitation Android (Esoko). Les communications sont rendues possibles grâce à une utilisation technologique minimaliste et parfois peu sophistiquée dans la plateforme Esoko pour permettre son utilisation le plus large possible, principalement par des fermiers, des commerçants et d'autres usagers du même ordre $^{14}$ (Salkovic, 2015, p. 4).

\footnotetext{
${ }^{13}$ www.Mxit.com

14 Traducion libre de : "Esoko is a simple communication tool that operates with any mixed modes of web and mobile applications but dominantly through SMS and quite recently on Android operating systems (Esoko). Communications are rendered and cultivated through minimalistic and sometimes unsophisticated technological use in the
} 
D'après Salkovic, l'utilisation d'Esoko est en train d'affecter la façon dont s'opèrent les communications et les relations interpersonnelles dans le Ghana rural et urbain. On peut donc en conclure que la particularité commune à toutes les applications comme Esoko ainsi qu'aux centaines d'autres de même nature créées partout en Afrique est qu'elles rendent compte du fait que leurs usagers sont "devenus producteurs, commentateurs et diffuseurs de contenus (données, messages, commentaires, photos, vidéos) »(Kalonji, Couve et Le Bot, 2016, p. 5), un fait confirmé par le développement rapide des startups en Afrique.

En effet, ces cinq dernières années, les startups, entreprises bâties autour des technologies de l'information et de la communication, ont connu une croissance significative en Afrique et provoqué une ruée des investisseurs vers le continent, au point où le GSMA $^{15}$ (2016) estime que « le mobile est devenu la plateforme de choix pour la création, la distribution et la consommation des solutions et des produits des services numériques innovants en Afrique ${ }^{16}$ » (GSMA, 2016, p. 24). Ces entreprises, qui sont aussi des applications développées localement, reposent sur des produits innovants et proposent des solutions aux problèmes de toute nature auxquelles les Africains doivent faire face dans leur vie quotidienne. II peut s'agir, par exemple, comme dans le cas de Sokowatch au Kenya, de donner la possibilité aux petits boutiquiers de passer commande auprès de gros fournisseurs par le moyen de SMS, puis de faire livrer ces commandes aux magasins désignés par des agents de la startup. II peut aussi s'agir, comme dans le cas de mPoultry, toujours au Kenya, de proposer aux fermiers africains, qui perdent chaque année jusqu'à $80 \%$ de leur stock de volaille en raison de couvoirs non surveillés, un dispositif qui permet de les alerter lorsqu'un problème est détecté (Hersman, 2012). On peut également citer le cas d'Intelworld en Ouganda, qui a développé une plateforme de commerce mobile appelée "Xente », qui permet aux clients du monde entier d'acheter des produits auprès des commerçants ougandais.

Esoko platform to encompass grass root level users comprising of mostly farmers, traders and other variables within the same scope ".

15 Global Systems for Mobile Communications.

16 Traduction libre de : "Mobile has emerged as the platform of choice for creating, distributing and consuming innovative digital solutions and services in Africa ». 
Changement technologique et sociabilité : les trois âges des communautés virtuelles africaines

II existe sur tout le continent des centaines d'autres initiatives du même ordre et leur nombre ne fait que croître au point de susciter un nouveau discours sur l'Afrique, un discours numérique dont les accents sont clairement afrofuturistes. Ce discours laisse entendre que l'Afrique pourrait devenir, demain, "le centre du développement technoscientifique ${ }^{17}$ " mondial (Yaszek, 2013, p. 9). Comme lors des premières années de diffusion d'Internet, de nouveaux mythes commencent ainsi à être créés autour du développement spectaculaire des startups en Afrique et certains y voient déjà les prémices d'une économie numérique africaine « inclusive et durable » (Rioux, 2018).

Soutenu par l'expansion des TIC, des startups et d'autres évènements de même nature, tels que le succès rencontré par le film Black Panther sur le continent, le discours afrofuturiste dont les origines remontent au début des années 1990 aux ÉtatsUnis (1994) trouve à s'exprimer dans de nombreux autres domaines, notamment le domaine artistique avec ce qu'on appelle aujourd'hui l'« art numérique africain " (Bristow, 2012). D'après Bristow, l'usage des TIC, entre autres l'Internet et le téléphone mobile, est en train d'influencer les « innovations esthétiques » et les pratiques socioculturelles en Afrique, notamment dans le domaine de la peinture et de la sculpture, mais aussi de la musique. Ainsi, le Tchado-Canadien Caleb Rimtobaye, aussi connu sous le nom de Daft Punk Africain, a abandonné le soul auquel il s'était initié à ses débuts pour créer un rythme afro-électronique aux accents futuristes. Sur son site Internet, cette nouvelle musique, l'AfrotroniX, est décrite comme « une création originale mêlant Djing, instruments live... et performances afro-urbaines dans un univers futuriste ${ }^{18}$ ». Dans une interview datée de juin 2017, Caleb Rimtobaye ajoute « qu'à la base, AfrotroniX est un concept avec une philosophie, l'Afrofuturisme qui essaye de présenter l'Afrique d'une manière différente, dans une autre figure qui est moins représentée » (MacDonald, 2017). L'existence d'un tel art musical, sculptural et visuel laisse supposer que les imaginaires africains sont en train d'être "colonisés " par la numérisation de la société et que de nouvelles représentations, associées à la technologie, sont en train de naître. Ces représentations ont pour particularité d'être tournées vers le futur, à la différence des conceptions

\footnotetext{
17 Traduction libre de : "The center of technoscientific development »..

${ }^{18}$ AfrotroniX.com/about
} 
traditionnelles qui étaient essentiellement «tournées vers le passé » (Bristow, 2012). Ces nouvelles représentations sont aussi portées par une nouvelle génération d'Africains dont les visions du monde, les pratiques et les comportements sont fortement marqués par des croyances et des crédos centrés sur la technologie. C'est la génération des milléniaux africains.

Rappelons à cet égard que les caractéristiques démographiques des usagers d'Internet sont à l'image des caractéristiques démographiques du continent, c'est-à-dire qu'il s'agit d'une population essentiellement jeune. Le dernier rapport des Nations Unies sur la population (UN DESA, 2017) rappelait à cet égard que l'âge médian en Afrique se situe autour de 19 ans, tandis qu'il est de plus de 41 ans en Europe. Plus de $40 \%$ des Africains ont moins de 15 ans et plus de $50 \%$ moins de 24 ans. On peut donc en tirer la conclusion que la majorité de la population africaine est constituée de Milléniaux, c'est-à-dire une génération qui «considère le téléphone portable comme l'objet technique en leur possession le plus important » (We are social, 2018). II s'agit d'une génération pour laquelle l'usage d'Internet tend à être intégré aux activités de la vie quotidienne dans toute leur diversité (politique, ludique, sociale, consumériste, etc.), à la différence des générations précédentes qui en faisaient des usages plus limités, essentiellement " engagés » ou " identitaires », c'est-à-dire des usages réduits aux aspects de sociabilité, notamment politique. En d'autres termes, la nouvelle génération dont il s'agit ici n'utilise pas Internet que pour communiquer. Le téléphone mobile est devenu pour elle un objet indispensable de la vie quotidienne pour presque tout ce qui concerne la vie de ses membres.

\section{Conclusion}

Comme nous l'avons montré, le rôle joué par les plateformes numériques et les réseaux sociaux sur la configuration des communautés virtuelles a connu une évolution rapide en Afrique. Cette évolution est avant tout le résultat des changements qui affectent la technologie, mais elle est aussi en partie due aux changements qui affectent la population d'utilisateurs du web et des attentes qui lui sont associées sur le continent. Les premières communautés virtuelles africaines s'étaient formées autour des listes de diffusion et étaient essentiellement 
Changement technologique et sociabilité : les trois âges des communautés virtuelles africaines

composées de membres de la diaspora qui avaient pour ambition de rétablir les liens de sociabilité entre individus originaires des mêmes pays ou nations, voire des mêmes ethnies ou tribus. Ces communautés ont pris, surtout en Afrique francophone, une dimension panafricaine sur les forums de discussion qui s'étaient créés au début des années 2000 en France et dont l'un des plus connus était Grioo.com. L'évolution rapide des technologies a cependant abouti à l'abandon progressif de ces forums de discussion et à la migration de leurs membres vers les réseaux sociaux et les services de messagerie. Les communautés virtuelles se sont alors transposées sur le sol africain et se sont " indigénisées ", considérablement multipliées et diversifiées alors même que l'utilisation des smartphones se généralisait, autorisant l'accès à l'Internet mobile et modifiant considérablement la population des utilisateurs du web et leur localisation. Des groupes plus divers, plus réduits, plus centrés sur des aspects sociétaux " mineurs", tels que la famille ou les amis, sont alors apparus en même temps que des organisations politiques locales, des communautés religieuses, des organisations de la société civile, des groupes professionnels ou des groupes d'étudiants, etc., créaient partout sur Facebook et sur d'autres plateformes numériques des sites web, des pages et des lieux de sociabilité numérique. Une troisième génération de communautés virtuelles a ensuite émergé, portée par les millénariaux africains, qui sont en train de créer des plateformes indigènes, pensées et opérées dans des perspectives proprement africaines.

La foi en la technologie constitue l'aspect central d'une grande partie de cette nouvelle génération dont les productions ludiques et artistiques témoignent d'une colonisation des imaginaires africains par les représentations liées aux technologies de l'information et de la communication. Certaines des questions que pose l'avènement de cette génération sont liées à la contradiction qui existe entre la " médiatisation » du présent et du futur de l'Afrique et les réalités vécues par les Africains dans des sociétés marquées par la pauvreté, les inégalités, la montée des violences interethniques, la croissance démographique et l'absence d'opportunités pour les jeunes. 


\section{Références}

Alzouma, G. (2008). Identities in a "Fragmegrated" World: Black cybercommunities and the French Integration System. Black and African Diaspora: An International Journal, 1(2), 201-214.

Alzouma, G. (2018). WhatsApp Village: A "Mediated" Family in Niger Republic. Dans J. A Dibakana et J. P. Missié (dir.), La Famille en Afrique (p. 425-448). Paris, France: L'Harmattan.

Anderson, B. (1991). Imagined Communities: Reflections on the Origin and Spread of Nationalism (Revised Edition). Londres, Royaume-Uni/New York, NY: Verso.

Bastian, M. (1999). Nationalism in a Virtual Space: Immigrant Nigerians on the Internet. West Africa Review, 1(1), 138-163.

Boutet, M. (2008). S'orienter dans les espaces sociaux en ligne. L'exemple d'un jeu. Sociologie du travail, 50(4), 447-470.

Bristow, T. (2012). We want the funk: What is Afrofuturism to the situation of digital arts in Africa?. Technoetic Arts: A Journal of Speculative Research, 10(1), 25-32.

Castells, M. (2000). Materials for an exploratory theory of the network society. British Journal of Sociology, 51(1), 5-24.

Chéneau-Loquay, A. (2010). Modes d'appropriation innovants du téléphone mobile en Afrique. Genève, Suisse: Union Internationale des Télécommunications (UIT).

Chigona, A. et Chigona, W. (2008). MXIT it up in the media: Media discourse analysis on a mobile instant messaging system. The Southern African Journal of Information and Communication, (9), 42-57.

Conte, B. (2001). La fracture numérique en Afrique. Bordeaux, France : Université Montesquieu.

Damome, E. (2012). Communautés médiatiques et dynamiques générationnelles à l'ère du numérique en Afrique subsaharienne. ILETI-Ş-IM, (16), 7-21.

Darkwa, O. et Mazibuko, F. (2000). Creating Virtual Learning Communities in Africa: Challenges and Prospects. First Monday, 5(5). Repéré à http://firstmonday.org/issues/issue5_ 5/darkwa/index.html 
Changement technologique et sociabilité : les trois âges des communautés virtuelles africaines

Dimaggio, P. et Hargittai, E. (2001). From the "digital divide" to "digital inequality": Studying Internet use as penetration increases. Working Papers 47. Princeton University, Woodrow Wilson School of Public and International Affairs, Center for Arts and Cultural Policy Studies.

Donsimoni, M. (2018). L'Afrique entre communautés traditionnelles et monde virtuel. Communication, technologie et développement, (5), 59-72.

Durando, D., Parker, M. et de la Harpe, R. (2007). Investigation into the usage of mobile instant messaging in tertiary education. Proceedings of the $16^{\text {th }}$ Conference of World Wide Web Application. New York, NY : Association for Computing Machinery.

Gabas, J. J. (dir.). (2004). Société numérique et développement en Afrique : usages et politiques publiques. Paris, France: GEMDEV.

Gensollen, M. (2006). La culture entre économie et écologie : l'exemple des communautés en ligne. Dans X. Greffe (dir.), Création et Diversité au miroir des industries culturelles. Actes des journées d'économie culturelle (p. 285-312). Repéré à https://www.cairn.info/creation-et-diversite-au-miroir-desindustries--9782110961976-p-285.html

Global Systems for Mobile Communications (GSMA). (2016). The Mobile Economy: Africa. Londres, Royaume-Uni: GSMA.

Hall, M. (1998). Africa connected. First Monday. Repéré à https://firstmonday.org/ojs/index.php/fm/article/view/627/548

Hersman, E. (2012). Mobilizing tech entrepreneurs in Africa (innovations case narrative: iHub. Innovations, 7(4), 59-67.

Internet World Stats. (2020). Internet Penetration in Africa 2020 Q1- March. Repéré à https://www.internetworldstats.com/ stats1.htm

Kalonji, C., Couve, P. et Le Bot, J. (2016). Citoyenneté, numérique : ce que l'Afrique prépare. Paris, France : Agence française de coopération médias.

Kiyindou, A. et Miéré Pélage, T. (2012). Réseaux virtuels, reconstruction du lien social et de l'identité dans la diaspora noire. Études de communication, (38).

https://doi.org/10.4000/edc.3425 
MacDonald, D. (2017). Afrotronix and the future sound of Africa. CultMTL magazine. Repéré à https://cultmtl.com/2017/06/ afrotronix/

Mansell, R. et When, U. (1998). Knowledge Societies: Information Technology for Sustainable Development. Oxford, Royaume-Uni : Oxford University Press.

Mercanti-Guérin, M. (2010). Analyse des réseaux sociaux et communautés en ligne : quelles applications en marketing ? Management \& Avenir, 32(2), 132-153.

Moran, B. (2000). Nigerian Identity Formations in the Usenet Newsgroup soc.culture.nigeria. Occasional Paper, N0 81. Édimbourg, Royaume-Uni: Centre of African Studies, Edinburgh University Press.

Ojo, T. (2005). ICTs and the construction of "imagined African communities" online. Dans B. Hipfl et T. Hug (dir.), Media Communities (p. 167-188). Munster, Allemagne : Waxmann Verlag.

Proulx, S. (2006). Communautés virtuelles : ce qui fait lien. Dans L. Poissant et M. Sénécal (dir.), Communautés virtuelles : penser et agir en réseau (p. 13-26). Québec, Canada: Presses de l'Université Laval.

Rheingold, H., (1994). Virtual Community. Londres, GrandeBretagne : Minerva.

Rioux, R. (2018, 23 mai). Et si l'Afrique réussissait, avec le numérique, à inventer une économie inclusive et durable? Le Monde.

Salkovic, C. (2015). Esoko and WhatsApp communication in Ghana: Mobile services such as Esoko and WhatsApp in reshaping interpersonal digital media communication in Ghana (Thèse de baccalauréat). Blekinge Institute of Technology.

Sangaré, B. (2017, 7 décembre). À Bamako, les derniers cybercafés font de la résistance. Le Monde. Repéré à https://www.lemonde.fr/afrique/article/2017/12/07/a-bamakoles-derniers-cybercafes-font-de-laresistance_5226274_3212.html 
Changement technologique et sociabilité : les trois âges des communautés virtuelles africaines

Sagna, O. (2006). La lutte contre la fracture numérique en Afrique : aller au-delà de l'accès aux infrastructures. Hermès, La revue, (45), 15-24.

Traoré, D. (2012). Entre profane et sacré : usages d'Internet et islam dans deux communautés musulmanes ouest-africaines à Montréal. Anthropologica, 44(1), 61-69.

United Nations Department of Economic and Social Affairs, Population Division (UN DESA). (2017). World Population Prospects: The 2017 Revision, Key Findings and Advance Tables. New York, NY : UN DESA.

United Nations Development Programme (UNDP). (2001). Making New Technologies Work for Human Development: Human Development Report 2001. New York, NY : UNDP.

Van Dijk, J. (2005). The Deepening Divide: Inequality in the Information Society. Londres, Royaume-Uni: Sage Publications.

We are social. (2018). Global Digital Report 2018. Repéré à https://digitalreport.wearesocial.com/

Witte, J. C. et Mannon, S. E. (2010). The Internet and social inequalities. New York, NY : Routledge.

Waverman, I., Meschi, M., et Fuss, M. (2005). The Impact of Telecoms on Economic Growth in Developing Countries, Africa: The Impact of Mobile Phones. Vodafone Policy Paper Series, 2(3), 10-24.

Yaszek, L. (2013). Race in Science Fiction: The Case of Afrofuturism. Repéré à http://virtual-sf.com/wpcontent/uploads/2013/08/Yaszek.pdf 